\title{
Quantum Optical Description of Phase Conjugation of Vector Vortex Beams in Stimulated Parametric Down-Conversion
}

\author{
A.G. de Oliveira $\odot,{ }^{1, *}$ N. Rubiano da Silva $\odot,{ }^{1}$ R. Medeiros de Araújo, ${ }^{1}$ P.H. Souto Ribeiro $\odot,{ }^{1, \dagger}$ and \\ S.P. Walborn $2,3,4$ \\ ${ }^{1}$ Departamento de Física, Universidade Federal de Santa Catarina, Florianóplis, Santa Catarina CEP \\ 88040-900, Brazil \\ ${ }^{2}$ Instituto de Física, Universidade Federal do Rio de Janeiro, Caixa Postal 68528, Rio de Janeiro, Rio de Janeiro \\ 21941-972, Brazil \\ ${ }^{3}$ Departamento de Física, Universidad de Concepción, Concepción 160-C, Chile \\ ${ }^{4}$ Millennium Institute for Research in Optics, Universidad de Concepción, Concepción, 160-C, Chile
}

(Received 4 May 2020; revised 9 June 2020; accepted 6 July 2020; published 18 August 2020)

\begin{abstract}
We present a quantum-optics approach for describing stimulated parametric down-conversion in the two-type-I crystal "sandwich" configuration, which allows for parametric interaction of vector vortex beams. We analyze the conditions for which phase conjugation of the seed vector beam occurs. We then use two strategies for defining generalized Stokes parameters to describe phase conjugation of vector vortex beams. These allow for geometrical representations, such as higher-order Poincaré spheres. Our results are useful for the description and design of stimulated and spontaneous parametric down-conversion experiments with vector vortex beams.
\end{abstract}

DOI: 10.1103/PhysRevApplied.14.024048

\section{INTRODUCTION}

Parametric interactions are nonlinear processes that describe energy exchange between oscillators [1]. In the optical regime, they may describe energy exchange between optical waves of different frequencies [2,3]. Among the optical parametric processes, much attention has been paid to parametric down-conversion, particularly in the spontaneous regime [4,5], known as SPDC. On the other hand, when one of the down-converted modes is seeded with a nonvacuum field such as a laser beam, stimulated parametric down-conversion (StimPDC) occurs [6]. StimPDC can be used to explore interesting processes such as phase conjugation [7], or produce nonclassical states of light [8-11].

In SPDC, the quantum state of the down-converted photons can be engineered using different combinations of sources. A widely employed example is the two-type-I crystal "sandwich" source, which was conceived to prepare postselection-free two-photon polarization states with a variable degree of entanglement [12]. StimPDC in this

\footnotetext{
*a.gustavo@posgrad.ufsc.br

†p.h.s.ribeiro@ufsc.br
}

Published by the American Physical Society under the terms of the Creative Commons Attribution 4.0 International license. Further distribution of this work must maintain attribution to the author(s) and the published article's title, journal citation, and DOI.
two-type-I crystal configuration has been used to efficiently probe the polarization entanglement of the source in the spontaneous regime [13].

More recently, it has been shown that the two-crystal sandwich source allows for StimPDC with vector vortex beams (VVBs) [14], which can be used both as the pump or the seed (stimulating) beam [15]. This is possible because the two crystals mediate the coherent interaction for two orthogonal polarization directions. Since these two orthogonal polarizations form a basis for the polarization state of paraxial beams, each polarization component of the pump and seed beams can participate in the down-conversion process. StimPDC with VVBs is an interesting subject due to the possibility of combining the broad range of applications of VVBs in fields such as optical communications [16-25], metrology [26], imaging [27-29], and optical simulation of quantum systems [30-33], together with features such as phase conjugation [15,34] and frequency conversion.

Phase conjugation of VVBs [15,35], in particular, is useful for applications analogous to those using a scalar phase-conjugated beam. For instance, correction of wave front distortion [36] and high-resolution imaging [37] are applications that can be directly generalized for the case of VVBs and propagation through anisotropic distorting media.

The purpose of this paper is to present a theoretical approach based on a quantum-optics formalism that allows for the description of two-type-I crystal StimPDC with 
VVBs, which to our knowledge has been lacking until now. Previous descriptions of StimPDC of transverse spatial modes [34,38,39] do not take into account the polarization degree of freedom (DOF), where much of the interesting and particular features lie. In Sec. III we first discuss StimPDC of plane waves with this source, focusing only on the polarization degree of freedom. We show how the entangled state that the source would produce in SPDC determines the polarization transformation from seed to idler beam in StimPDC. In Sec. IV we provide a theoretical quantum optics-based description of VVBs and then StimPDC with VVBs. We determine both the density operator and the detection probability (intensity) associated with the stimulated idler beam, showing that these correspond to the phase conjugation of the input seed VVB. In Secs. V and VI we describe phase conjugation of VVBs using generalized Stokes vector formalisms. The first approach focuses on cylindrically symmetric vector beams, and makes use of a geometrical description based on the higher-order Stokes parameters and the higher-order Poincaré sphere [40,41] to visualize the phase conjugation of VVBs. The second approach employs a general formalism analogous to two qubits that is applicable to any vector beam. Conclusions and avenues for future work are discussed in Sec. VII.

\section{PRELIMINARY CONCEPTS}

\section{A. Phase conjugation of polarization states and Stokes parameters}

Optical phase conjugation [36,42] is a phenomenon that becomes apparent when the complex representation is used for the electromagnetic field. The complex conjugate of the field is identical to it except for the minus sign in the phase term. Physically, this means that the phase conjugate of some light field is a replica that propagates backwards in time. As a result, linear and both spin and orbital angular momenta are reversed. In the following, we analyze the vector phase conjugation, which concerns only the spin angular momentum or polarization.

Let us consider a generic polarization pure state written with respect to the basis formed by $|H\rangle$ and $|V\rangle$, representing horizontal and vertical polarization states, respectively:

$$
|\theta, \phi\rangle=\cos \frac{\theta}{2}|H\rangle+e^{i \phi} \sin \frac{\theta}{2}|V\rangle .
$$

Mathematically, the conjugation of the above polarization state is defined as its complex conjugate:

$$
|\theta, \phi\rangle^{*}=\cos \frac{\theta}{2}|H\rangle+e^{-i \phi} \sin \frac{\theta}{2}|V\rangle=|\theta,-\phi\rangle .
$$

Note that, following this definition, the states $|H\rangle,|V\rangle,|D\rangle$, and $|A\rangle$ ( $D$ and $A$ standing for diagonal and antidiagonal polarizations, respectively) are their own conjugates, while the circularly polarized states $|R\rangle=(|H\rangle+i|V\rangle) / \sqrt{2}$ and $|L\rangle=(|H\rangle-i|V\rangle) / \sqrt{2}$ are conjugates of one another.

Conjugation of the polarization state of light may also be described in terms of the Stokes vector $\overrightarrow{\mathbf{S}}=$ $\left(S_{0}, S_{1}, S_{2}, S_{3}\right)^{T}$. The Stokes parameters $S_{i}$ are defined in the following way: $S_{0}=I$ is the total intensity of the beam; $S_{1}=I_{H}-I_{V}, S_{2}=I_{D}-I_{A}$, and $S_{3}=I_{R}-I_{L}$, where $I_{\varepsilon}$ stands for the remaining intensity after projection onto the state $|\varepsilon\rangle$. We may assume that the beam intensity is normalized to $1\left(S_{0}=1\right)$, so that the vector $\vec{S}=\left(S_{1}, S_{2}, S_{3}\right)^{T}$ is sufficient to characterize the polarization state. Under this condition, the degree of polarization is the Euclidean norm of $\vec{S}: p=\sqrt{S_{1}^{2}+S_{2}^{2}+S_{3}^{2}}$. For pure states, such as Eq. (1), the degree of polarization is maximum by definition, i.e. $p=1$.

Henceforth, we will refer to $\vec{S}$ as the Stokes vector, for simplicity. For state $|\theta, \phi\rangle$, the Stokes vector reads

$$
\vec{S}=(\cos \theta, \sin \theta \cos \phi, \sin \theta \sin \phi)^{T},
$$

while, for the conjugate state $|\theta, \phi\rangle^{*}$, we have

$$
\vec{S}^{*}=(\cos \theta, \sin \theta \cos \phi,-\sin \theta \sin \phi)^{T} .
$$

From Eq. (4), we can see that conjugation simply changes the sign of the $S_{3}$ (or $R / L$ ) component of the Stokes vector: $\vec{S}^{*}=\left(S_{1}, S_{2},-S_{3}\right)^{T}$. Therefore, in terms of the Poincaré sphere of polarization, which has the $|R\rangle(|L\rangle)$ state sitting on its north (south) pole, conjugation corresponds to a mirror reflection through the equatorial plane of the sphere [15].

\section{VECTOR PHASE CONJUGATION IN StimPDC AND BELL STATES}

The Bell basis of entangled polarization states is composed of four states. A SPDC source can be set in four different manners that creates each of the Bell states, or even produce superpositions of these states. Let us see how the vector phase conjugation appears in those scenarios.

Consider the two-crystal type-I SPDC source, as shown in Fig. 1(a), with an intense pump beam that has the polarization state $\left|\theta_{p}, \phi_{p}\right\rangle$. Let us assume that the fields are sufficiently monochromatic so that the coherence length is longer than the distance between crystals, and also that the fields are single spatial mode. These conditions, which can be achieved via pump engineering and filters, are necessary so that photons produced in either crystal are indistinguishable in all degrees of freedom besides the polarization. In this simple scenario, the effective SPDC Hamiltionian is proportional to $\cos \left(\theta_{p} / 2\right) \mathbf{a}_{V}^{\dagger} \mathbf{a}_{V}^{\dagger}+$ $\exp \left(i \phi_{p}\right) \sin \left(\theta_{p} / 2\right) \mathbf{a}_{H}^{\dagger} \mathbf{a}_{H}^{\dagger}$, where $a_{V(H)}^{\dagger}$ are creation operators in modes $V(H)$, which creates two photons with the same polarization. For SPDC, where the signal and idler 


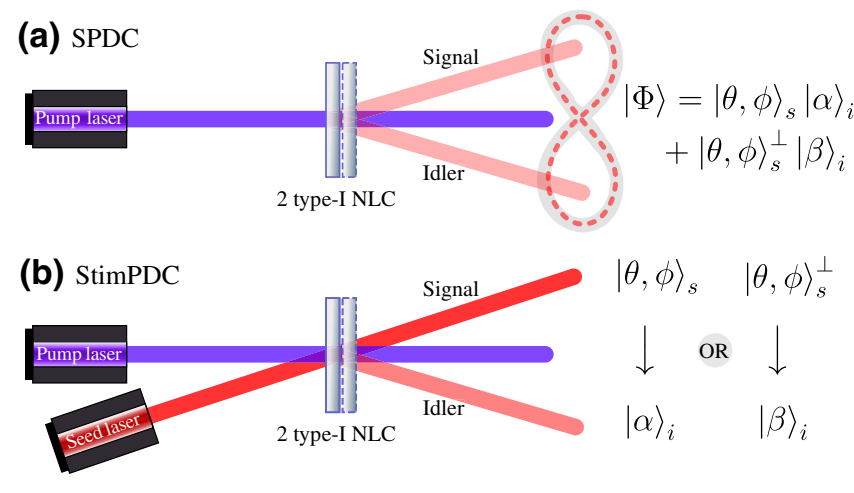

FIG. 1. (a) SPDC with two type-I crystals generating polarization entangled states. (b) StimPDC with two type-I crystals: the seed laser polarization determines the idler polarization. NLC denotes nonlinear crystal.

modes are initially in the vacuum state, it will produce the state

$$
|\Phi\rangle=\sin \frac{\theta_{p}}{2} e^{i \phi_{p}}|H\rangle_{s}|H\rangle_{i}+\cos \frac{\theta_{p}}{2}|V\rangle_{s}|V\rangle_{i},
$$

where it is clear that the pump beam polarization determines the entangled state. We can also consider a source that produce states of the form

$$
|\Psi\rangle=\sin \frac{\theta_{p}}{2} e^{i \phi_{p}}|H\rangle_{s}|V\rangle_{i}+\cos \frac{\theta_{p}}{2}|V\rangle_{s}|H\rangle_{i}=\mathbf{X}_{i}|\Phi\rangle,
$$

where $\mathrm{X}_{i}$ is the Pauli operator (half-wave plate at $45^{\circ}$ ) that changes $H \leftrightarrow V$. The same two-crystal type-I source with an additional half-wave plate can produce this state, or, alternatively, type-II crossed cones [43] and Sagnac source [44], for instance.

Now, let us rewrite the states of Eqs. (5) and (6) in a more general way using the following orthonormal basis for the signal photons: $\left\{|\theta, \phi\rangle_{s},|\theta, \phi\rangle_{s}^{\perp}\right\}$. Here $|\theta, \phi\rangle_{s}$ is defined as in Eq. (1) and

$$
|\theta, \phi\rangle_{s}^{\perp} \equiv|\theta-\pi, \phi\rangle_{s}=\sin \frac{\theta_{s}}{2}|H\rangle_{s}-e^{i \phi_{s}} \cos \frac{\theta_{s}}{2} \mid \eta_{s} .
$$

The results are

$$
|\Phi\rangle=|\theta, \phi\rangle_{s}|\alpha\rangle_{i}+|\theta, \phi\rangle_{s}^{\perp}|\beta\rangle_{i}
$$

and

$$
|\Psi\rangle=|\theta, \phi\rangle_{s} \mathrm{X}_{i}|\alpha\rangle_{i}+|\theta, \phi\rangle_{s}^{\perp} \mathrm{X}_{i}|\beta\rangle_{i},
$$

where

$$
\begin{aligned}
|\alpha\rangle_{i} & =\sin \frac{\theta_{p}}{2} \cos \frac{\theta_{s}}{2}|H\rangle_{i}+e^{-i\left(\phi_{s}-\phi_{p}\right)} \cos \frac{\theta_{p}}{2} \sin \frac{\theta_{s}}{2}|V\rangle_{i} \\
|\beta\rangle_{i} & =\sin \frac{\theta_{p}}{2} \sin \frac{\theta_{s}}{2}|H\rangle_{i}-e^{-i\left(\phi_{s}-\phi_{p}\right)} \cos \frac{\theta_{p}}{2} \cos \frac{\theta_{s}}{2}|V\rangle_{i} .
\end{aligned}
$$

Suppose now that we have StimPDC with the seed beam aligned to the signal arm, matched in path and frequency to the photon $s$. Moreover, we assume that the intensity of the seed beam is high enough so that the intensity of the idler beam is dominated by the stimulated process and the spontaneous emission contribution is negligible. In Fig. 1(b) we illustrate the StimPDC scheme. It has been shown that the stimulation of the signal beam can be interpreted as being equivalent to a state projection [45]. Therefore, we can see from Eqs. (8) and (9) that if the seed beam is prepared in the arbitrary polarization state $|\theta, \phi\rangle_{s}$ then the stimulated beam in the idler arm will leave the crystals in polarization state $|\alpha\rangle_{i}$ or $\mathrm{X}_{i}|\alpha\rangle_{i}$, depending on which type of source is used. These results may be summarized as $\mathrm{X}_{i}^{t}|\alpha\rangle_{i}$, where $t=0$ (1) when the source produces a state of type $\Phi(\Psi)$.

The effect of the Pauli operator $\mathrm{X}$ on an arbitrary Stokes vector $\vec{S}$ is given by $X\left[\left(S_{1}, S_{2}, S_{3}\right)^{T}\right]=\left(-S_{1}, S_{2},-S_{3}\right)^{T}$, since it interchanges both $H \leftrightarrow V$ and $R \leftrightarrow L$. In Ref. [15] the Stokes vector was derived for StimPDC with source $\Phi$ and a seed beam described by polarization state $|\alpha\rangle$. We can generalize these results to include the $\Psi$-type source. Namely, the Stokes vector of the stimulated beam can be written as

$$
\vec{S}_{i, t}=\frac{1}{2}\left(\begin{array}{c}
(-1)^{t}\left(S_{s 1}-S_{p 1}\right) \\
S_{p 2} S_{s 2}-S_{p 3} S_{s 3} \\
-(-1)^{t}\left(S_{s 2} S_{p 3}+S_{p 2} S_{s 3}\right)
\end{array}\right),
$$

where $S_{p j}$ and $S_{s j}$ are the Stokes parameters for the pump and seed beams, respectively, with $j=1,2,3$.

It is interesting to see what happens when the source is set to produce the four Bell states $\left|\Phi^{ \pm}\right\rangle$and $\left|\Psi^{ \pm}\right\rangle$. For StimPDC, the idler polarization for these four cases is

$$
\vec{S}_{i}^{\Phi^{+}}=\left(S_{s 1}, S_{s 2},-S_{s 3}\right),
$$

which is the usual vector phase conjugation;

$$
\vec{S}_{i}^{\Phi^{-}}=\left(S_{s 1},-S_{s 2}, S_{s 3}\right),
$$

which is "phase conjugation" but with respect to the $S_{2}$ axis; [15]

$$
\vec{S}_{i}^{\Psi^{+}}=\left(-S_{s 1}, S_{s 2}, S_{s 3}\right),
$$


which is "phase conjugation" but with respect to the $S_{1}$ axis; and

$$
\vec{S}_{i}^{\Psi^{-}}=\left(-S_{s 1},-S_{s 2},-S_{s 3}\right),
$$

which is a universal NOT operation, inverting the polarization state, as has been observed at the single-photon level $[46,47]$, where the fidelity of the NOT operation is reduced by the significant contribution of the spontaneous emission.

Recalling that phase conjugation depends on the basis chosen to represent the state, we interpret the results in Eqs. (12)-(14) as phase conjugation in different bases.

\section{StimPDC AND PHASE CONJUGATION OF VECTOR VORTEX BEAMS}

In this section we construct a more complete and more general theoretical description of StimPDC, which will allow for the description of StimPDC with VVBs. First, it will be useful to establish the quantum-optical description of coherent states and single-photon states in optical modes describing vector beams. The experimental scheme considered here is sketched in Fig. 2, where we show the usual scheme with one crystal and plane waves (for simplicity) in Fig. 2(a) and the two-type-I crystal scheme with VVBs in Fig. 2(b). At the end of this section, we show that this scheme allows for full phase conjugation of VVBs, i.e., that the idler beam is conjugated with respect to the seed beam's spatial mode and polarization.

\section{A. Quantum optical description of vector beams}

In classical optics, a paraxial, monochromatic vector beam can be written as

$$
\psi_{\mathrm{vb}}(\mathbf{q})=\gamma_{H} \mathbf{e}_{H} \psi_{H}(\mathbf{q})+\gamma_{V} \mathbf{e}_{V} \psi_{V}(\mathbf{q})
$$

where the $\mathbf{e}_{j}$ are unit polarization vectors in the $j=H, V$ directions, the $\gamma_{j}$ are complex numbers obeying $\left|\gamma_{H}\right|^{2}+$
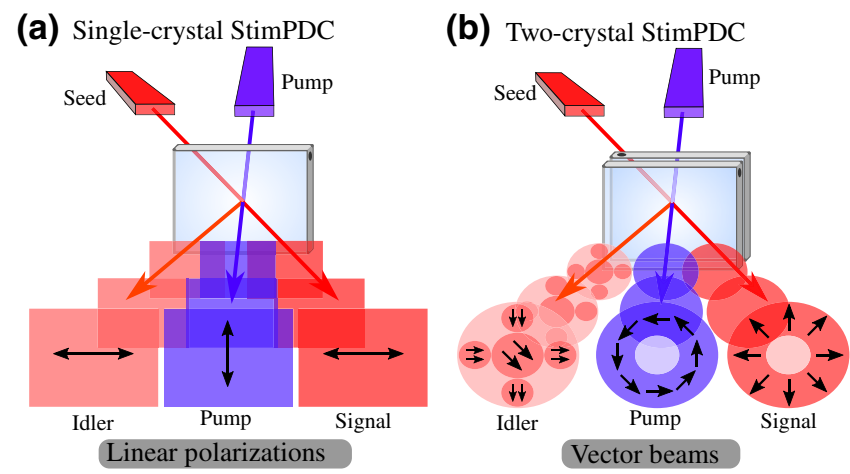

FIG. 2. Sketch of the StimPDC scheme for (a) the singlecrystal source and plane waves, (b) the two-type-I crystal source and vector beams.
$\left|\gamma_{V}\right|^{2}=1$, and the $\psi_{j}$ describe spatial modes in terms of transverse momentum coordinates $\mathbf{q}$.

Through quantization of Eq. (16), we can define the operator

$$
\mathrm{a}_{\mathrm{vb}}^{\dagger}=\gamma_{H} \mathrm{a}_{H, \psi_{H}}^{\dagger}+\gamma_{V} \mathrm{a}_{V, \psi_{V}}^{\dagger},
$$

where $a_{j, \psi_{j}}^{\dagger}$ creates a photon with polarization $j$ and transverse spatial mode $\psi_{j}$.

A coherent state with mean photon number $|\alpha|^{2}$, prepared in a vector beam mode, is denoted by $|\alpha\rangle_{\mathrm{vb}}$. This can be written in terms of creation operators as

$$
|\alpha\rangle_{\mathrm{vb}}=e^{-|\alpha|^{2} / 2} e^{\alpha \mathrm{a}_{\mathrm{vb}}^{\dagger}}|v a c\rangle
$$

Using the operator of Eq. (17), the state of Eq. (18) can be rewritten as

$$
\begin{aligned}
|\alpha\rangle_{\mathrm{vb}} & \left.=e^{-|\alpha|^{2} / 2} e^{\gamma_{H} \alpha \mathrm{a}_{H, \psi_{H}}^{\dagger}} e^{\gamma_{V} \alpha \mathrm{a}_{V, \psi_{V}}^{\dagger}} \mid \text { vac }\right\rangle \\
& =\left|\gamma_{H} \alpha\right\rangle_{H, \psi_{H}}\left|\gamma_{V} \alpha\right\rangle_{V, \psi_{V}},
\end{aligned}
$$

where in the second line we used $\left|\gamma_{H}\right|^{2}+\left|\gamma_{V}\right|^{2}=1$.

In a similar fashion, we can expand the transverse mode operators in terms of plane-wave modes,

$$
\mathrm{a}_{j, \psi_{j}}^{\dagger}=\sum_{\mathbf{q}} \psi_{j}(\mathbf{q}) \mathrm{a}_{j, \mathbf{q}} .
$$

In this way, a coherent state of the form $|\eta\rangle_{j, \psi_{j}}$ is

$$
\begin{aligned}
|\eta\rangle_{j, \psi_{j}} & =e^{-|\eta|^{2} / 2} e^{\eta \mathbf{a}_{j, \psi_{j}}^{\dagger}}|v a c\rangle \\
& =\prod_{\mathbf{q}} e^{-|\eta|^{2} / 2} e^{\eta \psi_{j}(\mathbf{q}) \mathbf{a}_{j, \mathbf{q}}^{\dagger}}|v a c\rangle \\
& =\prod_{\mathbf{q}}\left|\eta \psi_{j}(\mathbf{q})\right\rangle_{j, \mathbf{q}} .
\end{aligned}
$$

Finally, using Eq. (21), we can write the vector beam of Eq. (19) in terms of plane-wave modes as

$$
|\alpha\rangle_{\mathrm{vb}}=\prod_{\mathbf{q}}\left|\alpha \gamma_{H} \psi_{H}(\mathbf{q})\right\rangle_{H, \mathbf{q}}\left|\alpha \gamma_{V} \psi_{V}(\mathbf{q})\right\rangle_{V, \mathbf{q}} .
$$

Eq. (22) will be necessary for the next section.

Using the operator of Eq. (17), a single photon in a vector beam mode is

$$
|1\rangle_{\mathrm{vb}}=\mathrm{a}_{\mathrm{vb}}^{\dagger}|v a c\rangle=\gamma_{H}|1\rangle_{H, \psi_{H}}+\gamma_{V}|1\rangle_{V, \psi_{V}},
$$


which can be recast in terms of plane-wave modes using Eq. (20) as

$$
|1\rangle_{\mathrm{vb}}=\gamma_{H} \int d \mathbf{q} \psi_{H}(\mathbf{q})|1\rangle_{H, \mathbf{q}}+\gamma_{V} \int d \mathbf{q} \psi_{V}(\mathbf{q})|1\rangle_{V, \mathbf{q}},
$$

where we extend the summations to integrals. We can note the key difference between the vector beam composed of a single photon in Eq. (24) and the coherent state of Eq. (22) written in the plane-wave basis. While the latter is clearly a product state of all relevant modes, the single-photon state of Eq. (24) is an example of mode entanglement.

For comparison with the next section, let us note that the phase conjugate of this state is

$$
|1\rangle_{\mathrm{vb}}^{*}=\gamma_{H}^{*} \int d \mathbf{q} \psi_{H}^{*}(\mathbf{q})|1\rangle_{H, \mathbf{q}}+\gamma_{V}^{*} \int d \mathbf{q} \psi_{V}^{*}(\mathbf{q})|1\rangle_{V, \mathbf{q}} .
$$

\section{B. StimPDC of vector vortex beams}

Let us now turn to StimPDC of vector beams using a PDC source consisting of two type-I crystals as in Fig. 1(b). We assume that the monochromatic and paraxial approximations are valid for all fields, and that higherorder photon number terms (four or more SPDC photons) are negligible. We first consider a single down-conversion crystal that converts energy from a $H$ polarized pump field into down-converted fields with $V$ polarizations. As usual, we assume that the transverse width of the crystal is much larger than that of the transverse width of the pump beam. For our purposes, it is sufficient to consider the nonlinear interaction term described by $[48,49]$

$$
\iiint d \mathbf{q}_{p} d \mathbf{q}_{s} d \mathbf{q}_{i} F\left(\mathbf{q}_{s}, \mathbf{q}_{i}, \mathbf{q}_{p}\right) \mathbf{a}_{V, \mathbf{q}_{i}}^{\dagger} \mathbf{a}_{V, \mathbf{q}_{s}}^{\dagger} \mathbf{a}_{H, \mathbf{q}_{p}},
$$

where $F\left(\mathbf{q}_{s}, \mathbf{q}_{i}, \mathbf{q}_{p}\right)=\delta\left(\mathbf{q}_{s}+\mathbf{q}_{i}-\mathbf{q}_{p}\right) \operatorname{sinc}\left[\left(k_{i} \mathbf{q}_{s} / k_{s}-k_{s}\right.\right.$ $\left.\left.\mathbf{q}_{i} / k_{i}\right)^{2} d / 4 k_{p}\right]$ is the phase matching function, $d$ is the length of the nonlinear crystal, and $k_{s}, k_{i}$, and $k_{p}$ are the wave numbers of the signal, idler, and pump fields, respectively. Here we assume that the coupling between the polarization and spatial or spectral degrees of freedom can be ignored. This requires a thin crystal, or additional phase compensation using birrefringent elements [50]. Applying this operator to an intense pump beam that is described by a VVB $\left|\alpha_{p}\right\rangle_{\mathrm{vb}}$ as in Eq. (22), and noting that the coherent pump beam is an eigenstate of the interaction (26), allows us to write an effective Hamiltonian that acts only on the down-converted modes. Explicitly, applying Eq. (26) to Eq. (22) and tracing out the pump modes leads to

$$
\mathrm{H}_{H} \approx \gamma_{H} \iint d \mathbf{q}_{s} d \mathbf{q}_{i} \Psi_{H}\left(\mathbf{q}_{s}, \mathbf{q}_{i}\right) \mathbf{a}_{V, \mathbf{q}_{s}}^{\dagger} \mathbf{a}_{V, \mathbf{q}_{i}}^{\dagger}
$$

where

$$
\Psi_{H}\left(\mathbf{q}_{s}, \mathbf{q}_{i}\right)=\psi_{H}\left(\mathbf{q}_{s}+\mathbf{q}_{i}\right) \operatorname{sinc}\left[\frac{d}{4 k_{p}}\left(\frac{k_{i}}{k_{s}} \mathbf{q}_{s}-\frac{k_{s}}{k_{i}} \mathbf{q}_{i}\right)^{2}\right]
$$

and $\psi_{H}$ describes the angular spectrum of the pump beam.

In the two-crystal source, a second crystal converting the $V$ polarization component of the pump field to $H$-polarized down-converted fields is used, for which expressions analogous to Eqs. (27) and (28) can be written (with $H \leftrightarrow V$ ). Moreover, let us assume that these crystals are thin, with $d$ much smaller than the Rayleigh range of the pump beam, and placed very close together, as shown in Fig. 1(b). Under these conditions, the propagation effects between crystals can be ignored. With the above approximations, the effective Hamiltonian for parametric down-conversion with the two-crystal source and a VVB pump beam is

$$
\begin{aligned}
\mathrm{H} \approx & \gamma_{H} \iint d \mathbf{q}_{s} d \mathbf{q}_{i} \Psi_{H}\left(\mathbf{q}_{s}, \mathbf{q}_{i}\right) \mathbf{a}_{V, \mathbf{q}_{s}}^{\dagger} \mathrm{a}_{V, \mathbf{q}_{i}}^{\dagger} \\
& +\gamma_{V} \iint d \mathbf{q}_{s} d \mathbf{q}_{i} \Psi_{V}\left(\mathbf{q}_{s}, \mathbf{q}_{i}\right) \mathrm{a}_{H, \mathbf{q}_{s}}^{\dagger} \mathrm{a}_{H, \mathbf{q}_{i}}^{\dagger} .
\end{aligned}
$$

Here we recall that $\gamma_{H}$ and $\gamma_{V}$ are complex coefficients describing the horizontal and vertical polarization components of the pump vector beam, respectively. In StimPDC [38] with a vector beam as the seed signal beam, the PDC Hamiltonian of Eq. (29) is applied to the initial state $\left|\alpha_{s}\right\rangle_{\mathrm{sv}}|v a c\rangle$, where

$$
\left|\alpha_{s}\right\rangle_{\mathrm{sv}}=\prod_{\mathbf{q}_{s}}\left|\alpha_{s} \delta_{H} \phi_{H}\left(\mathbf{q}_{s}\right)\right\rangle_{H, \mathbf{q}_{s}}\left|\alpha_{s} \delta_{V} \phi_{V}\left(\mathbf{q}_{s}\right)\right\rangle_{V, \mathbf{q}_{s}}
$$

is the quantum optical description of the VVB of the form of Eq. (16), where $\delta_{H}$ and $\delta_{V}$ are the complex coefficients of each polarization component, $\left|\delta_{H}\right|^{2}+\left|\delta_{V}\right|^{2}=1$ and the subscript sv stands for seed VVB.

With this we can follow the usual steps and calculate the initial state using time-dependent perturbation theory $[48,49]$, resulting in

$$
\begin{aligned}
|\Psi\rangle \approx & \left|\alpha_{p}\right\rangle_{\mathrm{vb}}|v a c\rangle \\
& +C \gamma_{H} \iint d \mathbf{q}_{s} d \mathbf{q}_{i} \Psi_{H}\left(\mathbf{q}_{s}, \mathbf{q}_{i}\right)|1\rangle_{V, \mathbf{q}_{i}} \mathbf{a}_{V, \mathbf{q}_{s}}^{\dagger}\left|\alpha_{s}\right\rangle_{\mathrm{sv}} \\
& +C \gamma_{V} \iint d \mathbf{q}_{s} d \mathbf{q}_{i} \Psi_{V}\left(\mathbf{q}_{s}, \mathbf{q}_{i}\right)|1\rangle_{H, \mathbf{q}_{i}} \mathrm{a}_{H, \mathbf{q}_{s}}^{\dagger}\left|\alpha_{s}\right\rangle_{\mathrm{sv}}
\end{aligned}
$$

where $C \ll 1$ is a constant and $\left|C \alpha_{S}\right| \ll 1$ is required in order to make higher-order terms in the expansion negligible. This condition means that only a small fraction of pump photons is down-converted, even in the presence of 
the stimulating seed beam, which increases the emission in a given pair of modes.

From now on we assume that this state will be used to determine photon-counting probabilities or intensities, so that we can ignore the vacuum term and focus on the nonvacuum contribution. In each term of Eq. (31), the signal field is a multimode single-photon-added coherent state that, under proper conditions, can exhibit nonclassical $[8,51]$ and non-Gaussian [10] behavior. To proceed without loss of generality, we focus on the properties of the stimulated idler field by calculating its reduced density operator by tracing over the polarization and spatial state of the signal beam. This gives

$$
\begin{aligned}
\hat{\varrho}_{i} \approx \iiint \int d \mathbf{q}_{s} d \mathbf{q}_{i} d \mathbf{q}_{s}^{\prime} d \mathbf{q}_{i}^{\prime} \sum_{j, k=H, V} \gamma_{\bar{j}} \gamma_{\bar{k}}^{*}\left|j, \mathbf{q}_{i}\right\rangle\left\langle k, \mathbf{q}_{i}^{\prime}\right| \\
\times \Psi_{\bar{j}}\left(\mathbf{q}_{s}, \mathbf{q}_{i}\right) \Psi_{\bar{k}}^{*}\left(\mathbf{q}_{s}^{\prime}, \mathbf{q}_{i}^{\prime}\right)_{\mathrm{sv}}\left\langle\alpha_{s}\left|\mathbf{a}_{k, \mathbf{q}_{s}^{\prime}} \mathbf{a}_{j, \mathbf{q}_{s}}^{\dagger}\right| \alpha_{s}\right\rangle_{\mathrm{sv}},
\end{aligned}
$$

where $\bar{j}$ is the orthogonal polarization to $j$, and in an effort towards simplification we introduce the notation $\left\langle j, \mathbf{q}_{i}\right\rangle=$ $|1\rangle_{j, \mathbf{q}_{i}}$ for single-photon states.

Applying the commutation relation to the term

$$
{ }_{\mathrm{sv}}\left\langle\alpha_{s}\left|\mathbf{a}_{k, \mathbf{q}_{s}^{\prime}} \mathbf{a}_{j, \mathbf{q}_{s}}^{\dagger}\right| \alpha_{s}\right\rangle_{\mathrm{sv}}=\delta_{\mathbf{k}, \mathbf{q}_{\mathrm{s}}, \mathbf{j}, \mathbf{q}_{\mathrm{s}}^{\prime}}+{ }_{\mathrm{sv}}\left\langle\alpha_{s}\left|\mathbf{a}_{j, \mathbf{q}_{s}}^{\dagger} \mathbf{a}_{k, \mathbf{q}_{s}^{\prime}}\right| \alpha_{s}\right\rangle_{\mathrm{sv}}
$$

we obtain two terms, where the first term is related to the SPDC component and, using Eq. (30), the second term results in

$$
{ }_{\mathrm{sv}}\left\langle\alpha_{s}\left|\mathrm{a}_{j, \mathbf{q}_{s}}^{\dagger} \mathrm{a}_{k, \mathbf{q}_{s}^{\prime}}\right| \alpha_{s}\right\rangle_{\mathrm{sv}}=\left|\alpha_{s}\right|^{2} \delta_{j}^{*} \delta_{k} \phi_{j}^{*}\left(\mathbf{q}_{s}\right) \phi_{k}\left(\mathbf{q}_{s}^{\prime}\right) .
$$

The (unnormalized) density operator of the polarization and spatial degrees of freedom of the idler field is

$$
\hat{\varrho}_{i}=\hat{\varrho}_{i}^{\mathrm{SPDC}}+\left|\alpha_{s}\right|^{2} \hat{\varrho}_{i}^{\mathrm{Stim}},
$$

where the component arising from SPDC is

$$
\begin{aligned}
\hat{\varrho}_{i}^{\mathrm{SPDC}} \approx \iiint \int d \mathbf{q}_{s} d \mathbf{q}_{i} d \mathbf{q}_{s}^{\prime} d \mathbf{q}_{i}^{\prime} \sum_{j, k=H, V} \gamma_{\bar{j}} \gamma_{\bar{k}}^{*}\left|j, \mathbf{q}_{i}\right\rangle\left\langle k, \mathbf{q}_{i}^{\prime}\right| \\
\times \Psi_{\bar{j}}\left(\mathbf{q}_{s}, \mathbf{q}_{i}\right) \Psi_{\bar{k}}^{*}\left(\mathbf{q}_{s}^{\prime}, \mathbf{q}_{i}^{\prime}\right)
\end{aligned}
$$

and the component arising from StimPDC is

$$
\begin{aligned}
\hat{\varrho}_{i}^{\mathrm{Stim}} \approx \iint & \iint d \mathbf{q}_{s} d \mathbf{q}_{i} d \mathbf{q}_{s}^{\prime} d \mathbf{q}_{i}^{\prime} \sum_{j, k=H, V} \gamma_{\bar{j}} \gamma_{\bar{k}}^{*}\left|j, \mathbf{q}_{i}\right\rangle\left\langle k, \mathbf{q}_{i}^{\prime}\right| \\
& \times \delta_{j}^{*} \delta_{k} \Psi_{\bar{j}}\left(\mathbf{q}_{s}, \mathbf{q}_{i}\right) \Psi_{\bar{k}}^{*}\left(\mathbf{q}_{s}^{\prime}, \mathbf{q}_{i}^{\prime}\right) \phi_{j}^{*}\left(\mathbf{q}_{s}\right) \phi_{k}\left(\mathbf{q}_{s}^{\prime}\right) .
\end{aligned}
$$

Eq. (35) shows that the state of the idler beam is a convex sum of an entangled state component coming from the spontaneous emission and a separable state coming from the stimulated contribution. Therefore, the degree of entanglement depends on the stimulation strength given by $|\alpha|_{s}^{2}$.

To write the density operator in a more compact form, let us define the single-photon states

$$
\left|j, \Phi_{j}^{*}\right\rangle=\iint d \mathbf{q}_{s} d \mathbf{q}_{i} \Psi_{\bar{j}}\left(\mathbf{q}_{s}, \mathbf{q}_{i}\right) \phi_{j}^{*}\left(\mathbf{q}_{s}\right)\left|j, \mathbf{q}_{i}\right\rangle
$$

so that we can write the StimPDC component in terms of these states as

$$
\hat{\varrho}_{i}^{\text {Stim }}=\sum_{j, k=H, V} \gamma_{j} \gamma_{\bar{k}}^{*} \delta_{j}^{*} \delta_{k}\left|j, \Phi_{j}^{*}\right\rangle\left\langle k, \Phi_{k}^{*}\right|
$$

We note again that $\hat{\varrho}_{i}^{\text {Stim }}$ is not normalized. The idler states defined in Eq. (38) and used to write the StimPDC contribution are determined by the transverse mode functions of the seed VVB, as well as the pump beam through the two-photon amplitudes as defined in Eq. (28). This will be discussed in more detail below.

\section{Intensity distribution of the idler field}

Before discussing phase conjugation, let us briefly relate the above results to what is usually measured in the laboratory. In typical experiments, the VVB is observed by first performing a polarization projection, and then measuring the spatial distributions of each polarization state with a detector or camera. Let us consider this scenario, with projection onto state $|\theta, \phi\rangle$ defined in Eq. (1). We note that the total intensity can be obtained by simply summing the intensities for two orthogonal polarization projections. To determine the detection probability or intensity of the idler field, we can then calculate

$$
I_{\theta, \phi}\left(\mathbf{r}_{i}\right) \propto \operatorname{tr}\left[\mathrm{E}^{-}\left(\mathbf{r}_{i}, t\right) \mathrm{E}^{+}\left(\mathbf{r}_{i}, t\right)\left\langle\theta, \phi\left|\hat{\varrho}_{i}\right| \theta, \phi\right\rangle\right],
$$

which can be rewritten in terms of spontaneous and stimulated contributions using Eq. (35):

$$
I_{\theta, \phi}\left(\mathbf{r}_{i}\right)=I_{\theta, \phi}^{\mathrm{SPDC}}\left(\mathbf{r}_{i}\right)+\left|\alpha_{s}\right|^{2} I_{\theta, \phi}^{\mathrm{Stim}}\left(\mathbf{r}_{i}\right) .
$$

For paraxial propagation, the field operators corresponding to detection at position $\mathbf{r}=(x, y, z)$ (assuming free propagation from $z=0$ to $z$ ) can be written as

$$
\mathrm{E}^{+}(\mathbf{r}) \propto \int d \mathbf{q} e^{-i \mathbf{q} \cdot \mathbf{r}} e^{i q^{2} z / 2 k} \mathbf{a}_{\mathbf{q}}
$$


and we can calculate the StimPDC component

$$
\begin{aligned}
I_{\theta, \phi}^{\operatorname{Stim}}\left(\mathbf{r}_{i}\right)= & \left.\cos ^{2} \frac{\theta}{2}\left|\gamma_{V}\right|^{2}\left|\delta_{H}\right|^{2}\left|\mathrm{E}^{+}(\mathbf{r})\right| \Phi_{H}^{*}\right\rangle\left.\right|^{2} \\
& \left.+\sin ^{2} \frac{\theta}{2}\left|\gamma_{H}\right|^{2}\left|\delta_{V}\right|^{2}\left|\mathrm{E}^{+}(\mathbf{r})\right| \Phi_{V}^{*}\right\rangle\left.\right|^{2} \\
& +\frac{e^{i \phi}}{2} \sin \theta \gamma_{H}^{*} \gamma_{V} \delta_{H}^{*} \delta_{V}\left\langle\Phi_{V}^{*}\left|\mathrm{E}^{-}(\mathbf{r}) \mathrm{E}^{+}(\mathbf{r})\right| \Phi_{H}^{*}\right\rangle \\
& +\frac{e^{-i \phi}}{2} \sin \theta \gamma_{H} \gamma_{V}^{*} \delta_{H} \delta_{V}^{*}\left\langle\Phi_{H}^{*}\left|\mathrm{E}^{-}(\mathbf{r}) \mathrm{E}^{+}(\mathbf{r})\right| \Phi_{V}^{*}\right\rangle
\end{aligned}
$$

The first two terms are proportional to the intensity of the modes $\Phi_{H}$ and $\Phi_{V}$ at the transverse plane $z$ and the last two terms arise from interference between these two modes.

\section{Phase conjugation of the seed vector beam}

Let us determine conditions for which the idler field can be considered to be the phase conjugate of the VVB seed beam. First, let us consider an intense seed beam $\left|\alpha_{s}\right|^{2} \gg 1$, so that the SPDC component can be ignored. In this case, the output field corresponds to a pure state. As observed in Sec. III, the polarization of the pump beam plays a crucial role in phase conjugation of the polarization state of the seed beam. Following these results, choosing the pump beam as linear-diagonally polarized so that $\gamma_{H}=\gamma_{V}$, the idler field is described by

$$
\left|\psi_{i}\right\rangle \approx \delta_{H}^{*}\left|H, \Phi_{H}^{*}\right\rangle+\delta_{V}^{*}\left|V, \Phi_{V}^{*}\right\rangle .
$$

The presence of the complex conjugate of the polarization coefficients $\delta_{H(V)}^{*}$ shows that this degree of freedom is conjugated with respect to the seed beam. We can also see that the spatial modes $\Phi_{H(V)}^{*}$ defined in Eq. (38) depend on the phase conjugated amplitude of the seed. However, it still depends on the pump beam amplitude. Inspection of these states indicates that they correspond to phase conjugation of the transverse spatial modes in the limiting case in which the pump beam can be approximated as a plane wave, and the crystal is very thin, so that we have $\Psi_{\bar{j}}\left(\mathbf{q}_{s}, \mathbf{q}_{i}\right)=\delta\left(\mathbf{q}_{s}+\mathbf{q}_{i}\right)$ :

$$
\left|j, \Phi_{j}^{*}\right\rangle=\int d \mathbf{q}_{i} \phi_{j}^{*}\left(-\mathbf{q}_{i}\right)\left|j, \mathbf{q}_{i}\right\rangle \equiv\left\langle j, \phi_{j}^{*}\right\rangle .
$$

Here the idler mode is mirror reflected $\left(\mathbf{q}_{i} \rightarrow-\mathbf{q}_{i}\right)$, which can be absorbed into the definition of the coordinate system. Then, the idler field is described by

$$
\left|\psi_{i}\right\rangle \approx \delta_{H}^{*}\left|H, \phi_{H}^{*}\right\rangle+\delta_{V}^{*}\left|V, \phi_{V}^{*}\right\rangle .
$$

This is the phase conjugate of a single-photon vector beam of Eq. (25), showing that the idler photon of Eq. (46) is in a vector vortex mode that is exactly given by the phase conjugate of the seed VVB mode. The condition $\Psi_{\bar{j}}\left(\mathbf{q}_{s}, \mathbf{q}_{i}\right)=\delta\left(\mathbf{q}_{s}+\mathbf{q}_{i}\right)$ corresponds to a SPDC source that produces maximal spatial entanglement [52-54], so it is apparent that high-fidelity phase conjugation of the VVB is obtained when the source is capable of producing both high-quality polarization and spatial entanglement.

Let us further explore the phase conjugated output. The thin-crystal approximation is valid when the crystal length $d \ll z_{R}$ [55], where $z_{R}$ is the Rayleigh range of the pump laser. Under these conditions, the two-photon amplitudes are determined by the transverse spatial modes of the pump beam: $\Psi_{\bar{j}}\left(\mathbf{q}_{s}, \mathbf{q}_{i}\right)=\psi_{j}\left(\mathbf{q}_{s}+\mathbf{q}_{i}\right)$. Thus, the idler states become

$$
\left|j, \Phi_{j}^{*}\right\rangle=\int d \mathbf{q}_{i} \tau_{j}\left(\mathbf{q}_{i}\right)\left|j, \mathbf{q}_{i}\right\rangle
$$

with the mode functions given by the convolution

$$
\tau_{j}\left(\mathbf{q}_{i}\right)=\int d \mathbf{q}_{s} \psi_{\bar{j}}\left(\mathbf{q}_{s}+\mathbf{q}_{i}\right) \phi_{j}^{*}\left(\mathbf{q}_{s}\right) .
$$

Here we see that the conjugated spatial modes in Eq. (44) can be tailored by manipulating those of the pump beam. Under these conditions, the intensity distribution of the phase conjugated output $\left(\gamma_{H}=\gamma_{V}\right)$ after free propagation and projection onto the $|\theta, \phi\rangle$ polarization state can be obtained from Eq. (43), giving

$$
\begin{aligned}
I_{\theta, \phi}^{\operatorname{Stim}}\left(\mathbf{r}_{i}\right)= & \frac{1}{2} \cos ^{2} \frac{\theta}{2}\left|\delta_{H}\right|^{2}\left|\mathcal{F}_{H}\left(\boldsymbol{\rho}_{i}\right)\right|^{2} \\
& +\frac{1}{2} \sin ^{2} \frac{\theta}{2}\left|\delta_{V}\right|^{2}\left|\mathcal{F}_{V}\left(\boldsymbol{\rho}_{i}\right)\right|^{2} \\
& +\frac{e^{i \phi}}{4} \sin \theta \delta_{V} \delta_{H}^{*} \mathcal{F}_{V}^{*}\left(\rho_{i}\right) \mathcal{F}_{H}\left(\boldsymbol{\rho}_{i}\right) \\
& +\frac{e^{-i \phi}}{4} \sin \theta \delta_{H} \delta_{V}^{*} \mathcal{F}_{H}^{*}\left(\rho_{i}\right) \mathcal{F}_{V}\left(\rho_{i}\right),
\end{aligned}
$$

where $\boldsymbol{\rho}=(x, y)$,

$$
\mathcal{F}_{k}\left(\boldsymbol{\rho}_{\boldsymbol{i}}\right)=\int d \rho \mathcal{W}_{\bar{k}}(\boldsymbol{\rho}) \mathcal{U}_{k}^{*}(\boldsymbol{\rho}) \exp \left[i\left|\boldsymbol{\rho}_{i}-\boldsymbol{\rho}\right|^{2} \frac{k_{i}}{2 z}\right],
$$

and $\mathcal{W}_{\bar{k}}(\rho)$ and $\mathcal{U}_{k}^{*}(\rho)$ are the transverse mode profiles of the pump beam and seed beam, respectively.

In Fig. 3 we present the outcomes obtained using Eqs. (49) and (50) to produce numerical simulations for the intensity profiles and vector structure of simulated idler beams, for a few combinations of pump and seed beams. The dependence of the idler beam on both pump and seed parameters is clearly present.

\section{HIGHER-ORDER STOKES PARAMETERS AND HIGHER-ORDER POINCARÉ SPHERES}

Let us consider polarization states that are fully polarized. They are completely characterized by their Stokes 
(a)

$l=1(\operatorname{azim})$

Seed

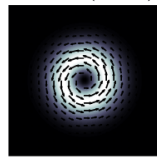

Idler

(b)

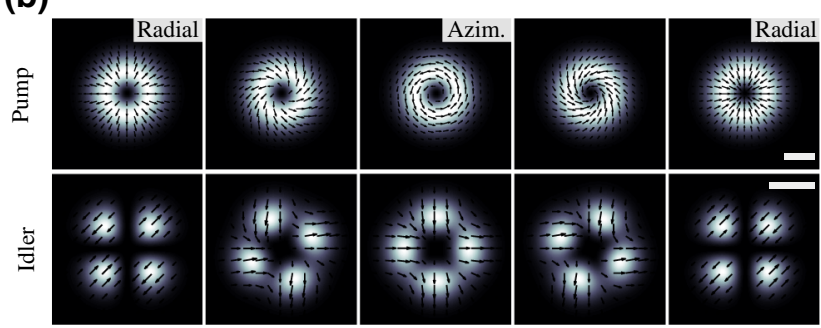

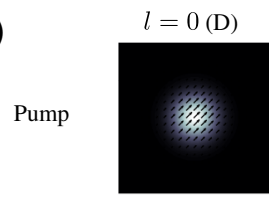

FIG. 3. Simulations of idler beams for a few combinations of pump and seed beams. (a) Transverse profiles of pump (top panel) and seed (middle panel) beams, and the corresponding simulated idler beam profile at the crystal plane (bottom panel). The seed is a vector vortex beam, while the pump takes linear and vector polarizations. (b) For a radial seed beam, the pump beam varies from radial (rad.) to azimuthal (azim.) with intermediate configurations. Both pump and calculated idler profiles are shown. For all subfigures, the color scale indicates the intensity, and the arrows, the polarization. The white scale bars represent unit beam radii (arb. unit).

parameters, which describe the content of polarization in each of the H/V, D/A, and R/L bases. These three parameters may be used as rectangular coordinates, locating the state at the surface of a sphere of unit radius, called the Poincaré sphere, with the R (L) state placed on its north (south) pole.

In previous works, we have drawn a geometrical picture of phase conjugation for scalar orbital angular momentum (OAM) modes [34] and for polarization states [15], using the Poincare sphere. Let us now turn to a convenient formalism for visualizing phase conjugation of a special case of VVBs using higher-order Stokes parameters (HOSPs) and higher-order Poincaré spheres (HOPSs).

\section{A. Cylindrical vector modes as two-dimensional systems}

Cylindrically symmetric vector (CSV) modes (propagation in the $z$ direction) have the form

$$
\left|\Psi_{\mathrm{CSV}}\right\rangle=\gamma_{1}|R,+l\rangle+\gamma_{2}|L,-l\rangle
$$

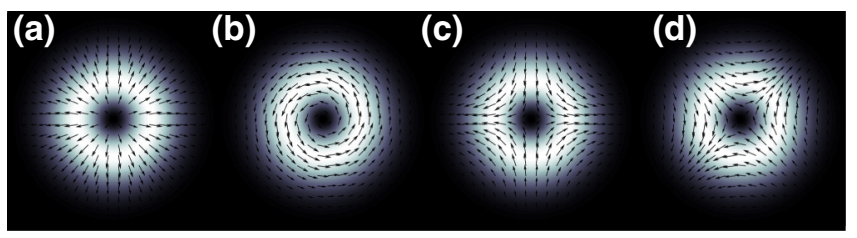

FIG. 4. Intensity and polarization profiles for $\gamma_{1}=\gamma_{2}$ and $\gamma_{1}=-\gamma_{2}$, respectively, for (a),(b) $l=+1$ and (c),(d) $l=-1$.

where $|R(L), l\rangle$ represents a state with spatial mode carrying a winding number $l=0, \pm 1, \pm 2, \ldots$ associated with OAM. The complex coefficients $\gamma_{1,2}$ are subject to the normalization condition $\left(\left|\gamma_{1}\right|^{2}+\left|\gamma_{2}\right|^{2}=1\right)$. In most cases the spatial component of the CSV modes is described by Laguerre-Gaussian transverse modes $\left(\mathrm{LG}_{l p}\right)$ with null radial number $p$ :

$$
\operatorname{LG}_{l 0}(\rho, \phi, z)=U_{|l|}(\rho, z) e^{-i l \phi} .
$$

Since $U_{|l|}(\rho, z)$ does not depend on the sign of the OAM, in a simplified picture the sets in Eq. (51) represent the azimuthal phase and polarization modes:

$$
\begin{aligned}
|R,+l\rangle & \sim e^{-i l \phi}\left(\mathbf{e}_{x}+i \mathbf{e}_{y}\right) \\
|L,-l\rangle & \sim e^{+i l \phi}\left(\mathbf{e}_{x}-i \mathbf{e}_{y}\right) .
\end{aligned}
$$

For $l \neq 0$, Eq. (51) defines two sets of states for each order $|l|$ depending on the sign of $l$. For example, for $|l|=1$, the set for $l=+1$ consists of rotationally symmetric vector modes such as the radial $\left(\gamma_{1}=\gamma_{2}\right)$ and azimuthal $\left(\gamma_{1}=-\gamma_{2}\right)$ modes of Figs. 4(a) and 4(b). This set of modes are especially important for tighter beam focusing [28] and alignment-free optical communications [17]. In Figs. 4(c) and 4(d) we represent two antivortex modes $\left(\gamma_{1}=\gamma_{2}\right.$ and $\gamma_{1}=-\gamma_{2}$, respectively) contained in the $l=-1$ set.

One may note that, for a given $l$, the CSV modes contained in the set can be spanned by a two-dimensional basis

$$
\left\{\left|0_{l}\right\rangle=|R, l\rangle,\left|1_{l}\right\rangle=|L,-l\rangle\right\},
$$

so that, ignoring the spatial amplitude $U_{|l|}$,

$$
\left|\psi_{\mathrm{CSV}}\right\rangle=\cos (\theta / 2)\left|0_{l}\right\rangle+e^{i \varphi} \sin (\theta / 2)\left|1_{l}\right\rangle
$$

represents a general CSV mode of the set. It is important to note that we need two distinct two-dimensional spaces to describe all the CSV modes of a given order $|l|$, depending on the sign of $l$. Of course, other bases can be constructed by linear combinations of the basis states of Eq. (54).

\section{B. HOSPs and HOPSs}

In the formalism of density matrices, two-dimensional CSV states of a given $l$ can be spanned in terms of Pauli 
matrices $\left(\hat{\sigma}_{1} \equiv \hat{\sigma}_{z}, \hat{\sigma}_{2} \equiv \hat{\sigma}_{x}\right.$ and $\left.\hat{\sigma}_{3} \equiv \hat{\sigma}_{y}\right)$, i.e.,

$$
\hat{\rho}^{l}=\frac{1}{2} \sum_{n=0}^{3} S_{n}^{l} \hat{\sigma}_{n},
$$

weighted by the so-called higher-order Stokes parameters $[40,41]$

$$
S_{n}^{l}=\operatorname{tr}\left[\hat{\rho}^{l} \hat{\sigma}_{n}\right]
$$

which reduce to the usual Stokes parameters for $l=0$. In terms of basis states of Eq. (54), these parameters are

$$
\begin{aligned}
& S_{0}^{l}=\left|\left\langle 0_{l} \mid \psi_{\mathrm{CSV}}\right\rangle\right|^{2}+\left|\left\langle 1_{l} \mid \psi_{\mathrm{CSV}}\right\rangle\right|^{2}, \\
& S_{1}^{l}=2 \operatorname{Re}\left(\left\langle 0_{l} \mid \psi_{\mathrm{CSV}}\right\rangle^{*}\left\langle 1_{l} \mid \psi_{\mathrm{CSV}}\right\rangle\right), \\
& S_{2}^{l}=2 \operatorname{Im}\left(\left\langle 0_{l} \mid \psi_{\mathrm{CSV}}\right\rangle^{*}\left\langle 1_{l} \mid \psi_{\mathrm{CSV}}\right\rangle\right), \\
& S_{3}^{l}=\left|\left\langle 0_{l} \mid \psi_{\mathrm{CSV}}\right\rangle\right|^{2}-\left|\left\langle 1_{l} \mid \psi_{\mathrm{CSV}}\right\rangle\right|^{2},
\end{aligned}
$$

and, as usual, the degree of polarization is $p=$ $\sqrt{\left(S_{1}^{l}\right)^{2}+\left(S_{2}^{l}\right)^{2}+\left(S_{3}^{l}\right)^{2}} \leq 1$, with the equality holding only for completely polarized light.

The mapping of CSV modes in two-dimensional spaces admits a geometrical representation in terms of HOPSs $[40,41,56]$, constructed by using $S_{1}^{l}, S_{2}^{l}$, and $S_{3}^{l}$ as Cartesian coordinates, or, equivalently, by spherical coordinates defined by

$$
r=p, \quad \theta=\cos ^{-1}\left(\frac{S_{3}^{l}}{S_{0}^{l}}\right), \quad \varphi=\tan ^{-1}\left(\frac{S_{2}^{l}}{S_{1}^{l}}\right) .
$$

Note that the HOPS representation is simply the Poincaré sphere representation for states of Eqs. (55) and (56). Of course, two spheres are needed to represent the two sets of modes of a given order $|l|$. In Fig. 5 the representation for usual states with $|l|=1$ is shown.

\section{Phase conjugation on the HOPS}

Let us now investigate the phase conjugation of CSV modes and how we can interpret it geometrically on the HOPS. Since the operation performed by our two-crystal StimPDC source depends on the properties of the pump beam, let us consider the more usual case where the phase conjugation implies in changing $l \rightarrow-l[34,39]$ and $R \leftrightarrow$ $L$ [15]. We see from the expressions in Eqs. (53) and (55) that

$$
\begin{aligned}
& \left|0_{l}\right\rangle^{*}=e^{+i l \phi}\left(\mathbf{e}_{x}-i \mathbf{e}_{y}\right)=\left|1_{l}\right\rangle, \\
& \left|1_{l}\right\rangle^{*}=e^{-i l \phi}\left(\mathbf{e}_{x}+i \mathbf{e}_{y}\right)=\left|0_{l}\right\rangle .
\end{aligned}
$$

(a)

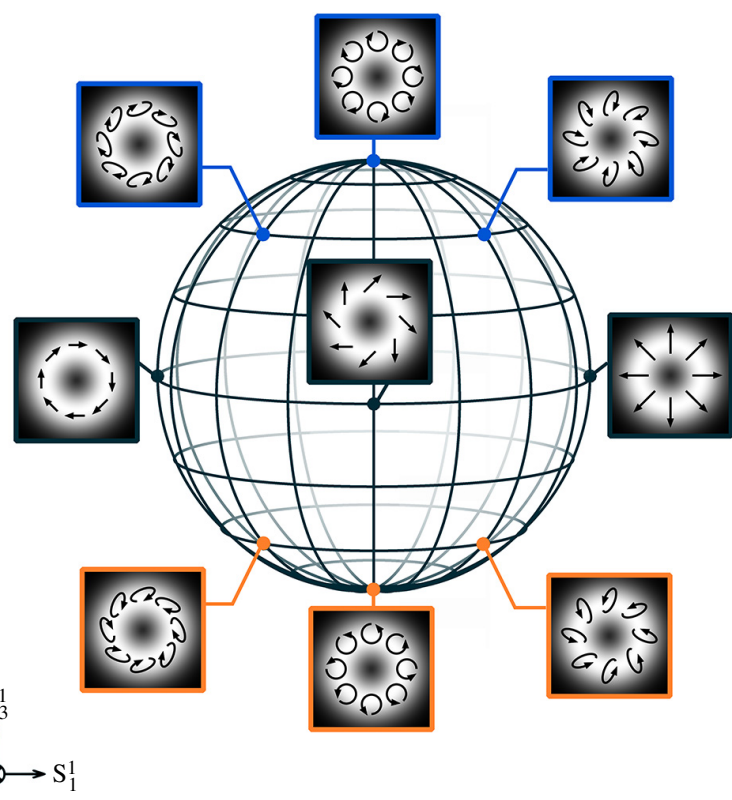

(b)

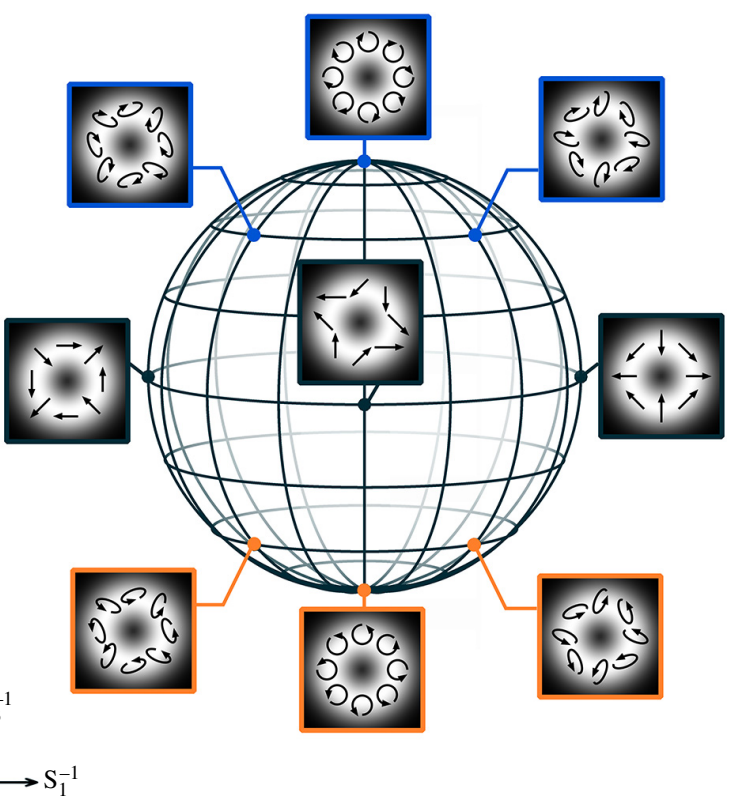

FIG. 5. Higher-order Poincaré spheres for (a) $l=+1$ and (b) $l=-1$.

Equation (60) shows that the conjugate counterpart of a general CSV beam of Eq. (55) is given by

$$
\left|\psi_{\mathrm{CSV}}\right\rangle^{*}=\cos (\theta / 2)\left|1_{l}\right\rangle+e^{-i \varphi} \sin (\theta / 2)\left|0_{l}\right\rangle,
$$

which in turn can be rewritten as

$$
\left|\psi_{\mathrm{CSV}}\right\rangle^{*}=\cos \left(\frac{\pi-\theta}{2}\right)\left|0_{l}\right\rangle+e^{i \varphi} \sin \left(\frac{\pi-\theta}{2}\right)\left|1_{l}\right\rangle .
$$


Equation (62) shows that conjugate beams are connected by reflection across the equatorial plane of the HOPS (which can be seen as changing $S_{3}^{l} \rightarrow-S_{3}^{l}$ ), which agrees with the $l=0$ case. Thus, we see that conjugate counterparts belong to the same HOPS as the original CSV, illustrating that vector beam conjugation does not alter the nature of the correlations between spatial and polarization degrees of freedom, since changing spheres would imply changing correlation to anticorrelation and vice versa. Moreover, we see that radially polarized beams [28] or rotationally invariant $(l=1)$ [17] beams do not lose their interesting properties under phase conjugation.

\section{PHASE CONJUGATION OF VECTOR BEAMS: GENERALIZED STOKES PARAMETERS}

While describing CSV modes in two-dimensional spaces gives us a practical geometrical tool for representing the modes and interpreting vector phase conjugation, we cannot represent all possible CSV states for a given order in this way, since we cannot represent a generic statistical mixture or even a superposition of modes in different two-dimensional subspaces as defined in the previous two sections. Moreover, we can also create a vector beam that is a superposition of modes with different values of $|l|$. For a general description of phase conjugation of VVBs in terms of Stokes parameters, we employ a description inspired by the generalized Bloch vector for two qubits. First, since the polarization DOF is two dimensional, Schmidt decomposition of a vector beam can always be represented by a $2 \times 2$ system, in analogy with the case of two qubits.

For a two-qubit system, the density operator can be spanned as [57]

$$
\hat{\rho}=\frac{1}{4} \sum_{i, j=0}^{3} S_{i j}\left(\hat{\sigma}_{i} \otimes \hat{\sigma}_{j}\right),
$$

which is equivalent to Eq. (56) generalized for a fourdimensional system.

The first qubit corresponds to the polarization DOF and the second to the transverse spatial mode. In this way, we can write the Stokes matrix as

$$
\mathbb{S}=\left(\begin{array}{cccc}
1 & s_{01} & s_{02} & s_{03} \\
s_{10} & s_{11} & s_{12} & s_{13} \\
s_{20} & s_{21} & s_{22} & s_{23} \\
s_{30} & s_{31} & s_{32} & s_{33}
\end{array}\right)
$$

where we used $s_{00}=1$, corresponding to normalized components. Note that $s_{0 j}$ and $s_{j 0}(j=1,2,3)$ are the Stokes vector components of the individual qubits, while the $s_{j k}$ components contain the correlations.

Using the representations $\hat{\sigma}_{1} \equiv \hat{\sigma}_{z}, \hat{\sigma}_{2} \equiv \hat{\sigma}_{x}$, and $\hat{\sigma}_{3} \equiv$ $\hat{\sigma}_{y}$, as in the previous section, phase conjugation of the vector beam changes the sign of the third component of both (a)

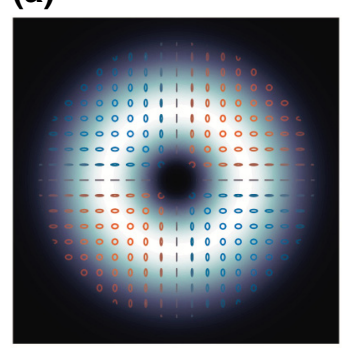

(b)

(c) $\mathrm{S}_{3}$

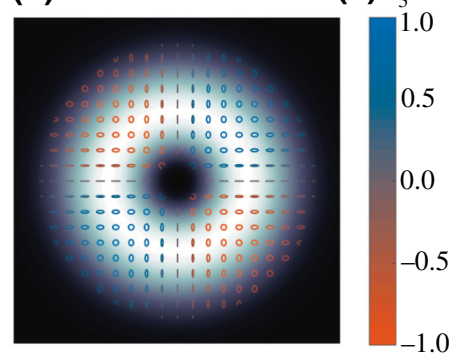

FIG. 6. (a) Anisotropic CSV beam and (b) phase conjugate beam. (c) Color bar for the $S_{3}$ Stokes parameter for polarization.

qubits; thus,

$$
\mathbb{S}^{*}=\left(\begin{array}{cccc}
1 & s_{01} & s_{02} & -s_{03} \\
s_{10} & s_{11} & s_{12} & -s_{13} \\
s_{20} & s_{21} & s_{22} & -s_{23} \\
-s_{30} & -s_{31} & -s_{32} & s_{33}
\end{array}\right)
$$

For example, in Ref. [15] an anisotropic vector beam was produced, which can be represented by

$$
|\psi\rangle_{\text {aniso }}=|D,+| l|\rangle+|A,-| l|\rangle,
$$

where $|D(A)\rangle=[|H\rangle+(-)|V\rangle] / \sqrt{2}$ are diagonal (antidiagonal) linear polarization states. In Fig. 6(a) we can see a plot of this beam, which can also be represented as

$$
\mathbb{S}_{\text {aniso }}=\left(\begin{array}{llll}
1 & 0 & 0 & 0 \\
0 & 1 & 0 & 0 \\
0 & 0 & 0 & 1 \\
0 & 0 & 1 & 0
\end{array}\right)
$$

and the Stokes matrix of the conjugate beam [(Fig. 6(b)] is

$$
\mathbb{S}_{\text {aniso }}^{*}=\left(\begin{array}{cccc}
1 & 0 & 0 & 0 \\
0 & 1 & 0 & 0 \\
0 & 0 & 0 & -1 \\
0 & 0 & -1 & 0
\end{array}\right)
$$

which agrees with the experimental results in Ref. [15].

\section{CONCLUSION}

In conclusion, we introduce a theoretical approach for the description of StimPDC including the use of vector vortex beams, which is based on a quantum-optics formalism. In addition to general expressions showing the interplay between the polarization and spatial degrees of freedom of the pump and seed (signal) beam, we describe the conditions for which the stimulated idler field is the phase conjugate of the input seed beam. We also make use of higher-order Stokes parameters and Poincaré spheres 
to provide a geometrical interpretation for phase conjugation of cylindrically symmetric vortex beams in StimPDC, and provide a general Stokes matrix description of phase conjugation that is applicable to any VVB.

The results presented here are a contribution to the field of nonlinear optics with vectorial and structured light. We note that recent work has studied polarization control and transverse mode mixing in up-conversion with single typeII crystals [58,59], as well as up-conversion of VVBs using a type-II crystal entanglement source [60]. We believe that both the combination of StimPDC and VVBs and our theoretical approach are very useful for a broad range of applications and experimental investigations. A possible application of our results is the enhanced analysis of SPDC sources of entangled vortex modes or hyperentangled states involving polarization and transverse modes similar to that shown in Ref. [61], for instance, following the lines of the fast and efficient entangled state tomography using StimPDC, demonstrated in Ref. [13]. In a similar way, our approach could be useful to improve the characterization of symmetry properties of nonlinear crystals using structured beams, as shown in Ref. [62], adding StimPDC as a resource to speed up the measurements. The nonclassical properties of the multimode output of the signal beam could also be explored. Moreover, phase conjugation of VVBs could be used to mediate detrimental propagation effects due to atmospheric turbulence and other causes. An experiment exploring the capabilities of StimPDC and VVBs is currently in progress in our laboratory.

\section{ACKNOWLEDGMENTS}

The authors thank the Brazilian Agencies CNPq, FAPESC, FAPERJ (Grants No. E- 26/010.002997/2014 and No. E-26/202.7890/2017), and the Brazilian National Institute of Science and Technology of Quantum Information (INCT/IQ). This study was funded in part by the Coordenação de Aperfeiçoamento de Pessoal de Nível Superior-Brasil (CAPES) - Finance Code 001. S.P.W. received support from the Chilean Fondo Nacional de Desarrollo Científico y Tecnológico (FONDECYT) (Grant No. 1200266) and Millennium Institute for Research in Optics.

[1] A. E. Siegman, Obtaining the equations of motion for parametrically coupled oscillators or waves, Proceedings of the IEEE 54, 756 (1966).

[2] A. Yariv, Quantum theory for parametric interactions of light and hypersound, IEEE. J. Quantum. Electron. 1, 28 (1965).

[3] A. Yariv and W. Louisell, 5A2 - theory of the optical parametric oscillator, IEEE. J. Quantum. Electron. 2, 418 (1966).
[4] D. Burnham and D. Weinberg, Observation of Simultaneity in Parametric Productions of Optical Photon Pairs, Phys. Rev. Lett. 25, 84 (1970).

[5] L. Mandel and E. Wolf, Optical Coherence and Quantum Optics (Cambridge University Press, New York, 1995).

[6] L. Wang, X. Zou, and L. Mandel, Observation of induced coherence in two-photon downconversion, J. Opt. Soc. Am. B 8, 978 (1991).

[7] P. H. Souto Ribeiro, D. P. Caetano, M. P. Almeida, J. A. Huguenin, B. Coutinho dos Santos, and A. Z. Khoury, Observation of Image Transfer and Phase Conjugation in Stimulated Down-Conversion, Phys. Rev. Lett. 87, 133602 (2001).

[8] A. Zavatta, S. Viciani, and M. Bellini, Quantum-toclassical transition with single-photon-added coherent states of light, Science 306, 660 (2004).

[9] A. Kolkiran and G. S. Agarwal, Quantum interferometry using coherent beam stimulated parametric downconversion, Opt. Express 16, 6479 (2008).

[10] M. Barbieri, N. Spagnolo, M. G. Genoni, F. Ferreyrol, R. Blandino, M. G. A. Paris, P. Grangier, and R. Tualle-Brouri, Non-Gaussianity of quantum states: An experimental test on single-photon-added coherent states, Phys. Rev. A 82, 063833 (2010).

[11] T. Kiesel, W. Vogel, M. Bellini, and A. Zavatta, Nonclassicality quasiprobability of single-photon-added thermal states, Phys. Rev. A 83, 032116 (2011).

[12] P. G. Kwiat, E. Waks, A. G. White, I. Appelbaum, and P. H. Eberhard, Ultrabright source of polarization-entangled photons, Phys. Rev. A. 60, R773 (1999).

[13] L. A. Rozema, C. Wang, D. H. Mahler, A. Hayat, A. M. Steinberg, J. E. Sipe, and M. Liscidini, Characterizing an entangled-photon source with classical detectors and measurements, Optica 2, 430 (2015).

[14] C. Rosales-Guzmán, B. Ndagano, and A. Forbes, A review of complex vector light fields and their applications, J. Opt. 20, 123001 (2018).

[15] A. G. de Oliveira, M. F. Z. Arruda, W. C. Soares, S. P. Walborn, R. M. Gomes, R. Medeiros de Araújo, and P. H. Souto Ribeiro, Real-time phase conjugation of vector vortex beams, ACS Photonics 7, 249 (2020).

[16] C. E. R. Souza, C. V. S. Borges, A. Z. Khoury, J. A. O. Huguenin, L. Aolita, and S. P. Walborn, Quantum key distribution without a shared reference frame, Phys. Rev. A 77, 032345 (2008).

[17] V. D’Ambrosio, E. Nagali, S. P. Walborn, L. Aolita, S. Slussarenko, L. Marrucci, and F. Sciarrino, Complete experimental toolbox for alignment-free quantum communication, Nat. Commun. 3, 961 (2012).

[18] O. J. Farías, V. D’Ambrosio, C. Taballione, F. Bisesto, S. Slussarenko, L. Aolita, L. Marrucci, S. P. Walborn, and F. Sciarrino, Resilience of hybrid optical angular momentum qubits to turbulence, Sci. Rep. 5, 8424 (2015).

[19] Y. Zhao and J. Wang, High-base vector beam encoding/decoding for visible-light communications, Opt. Lett. 40, 4843 (2015).

[20] G. Milione, T. A. Nguyen, J. Leach, D. A. Nolan, and R. R. Alfano, Using the nonseparability of vector beams to encode information for optical communication, Opt. Lett. 40, 4887 (2015). 
[21] G. Milione, M. P. J. Lavery, H. Huang, Y. Ren, G. Xie, T. A. Nguyen, E. Karimi, L. Marrucci, D. A. Nolan, and R. R. Alfano, et al., $4 \times 20 \mathrm{Gbit} / \mathrm{s}$ mode division multiplexing over free space using vector modes and a q-plate mode (de)multiplexer, Opt. Lett. 40, 1980 (2015).

[22] B. Ndagano, R. Brüning, M. McLaren, M. Duparré, and A. Forbes, Fiber propagation of vector modes, Opt. Express 23, 17330 (2015).

[23] J. Zhang, F. Li, J. Li, Y. Feng, and Z. Li, 120 Gbit/s $2 \times 2$ vector- modes-division- multiplexing DD-OFDM-32QAM free-space transmission, IEEE Photon. J. 8, 7907008 (2016).

[24] P. Li, B. Wang, and X. Zhang, High-dimensional encoding based on classical nonseparability, Opt. Express 24, 15143 (2016).

[25] B. Ndagano, I. Nape, M. A. Cos, C. Rosales-Guzman, and A. Forbes, Creation and detection of vector vortex modes for classical and quantum communication, J. Lightwave Tech. 36, 292 (2018).

[26] V. D’Ambrosio, N. Spagnolo, L. D. Re, S. Slussarenko, Y. Li, L. C. Kwek, L. Marrucci, S. P. Walborn, L. Aolita, and F. Sciarrino, Photonic polarization gears for ultra-sensitive angular measurements, Nat. Commun. 4, 2432 (2013).

[27] D. P. Biss, K. S. Youngworth, and T. G. Brown, Dark-field imaging with cylindrical-vector beams, Appl. Opt. 45, 470 (2006).

[28] Q. Zhan, Cylindrical vector beams: From mathematical concepts to applications, Adv. Opt. Photon. 1, 1 (2009).

[29] M. Yoshida, Y. Kozawa, and S. Sato, Subtraction imaging by the combination of higher-order vector beams for enhanced spatial resolution, Opt. Lett. 44, 883 (2019).

[30] A. N. de Oliveira, S. P. Walborn, and C. H. Monken, Implementation of the Deutsch algorithm using polarization and transverse modes, Journal of Optics B: Quantum and Semiclassical Optics 7, 288 (2005).

[31] C. E. R. Souza, J. A. O. Huguenin, P. Milman, and A. Z. Khoury, Topological Phase for Spin-Orbit Transformations on a Laser Beam, Phys. Rev. Lett. 99, 160401 (2007).

[32] C. V. S. Borges, M. Hor-Meyll, J. A. O. Huguenin, and A. Z. Khoury, Bell-like inequality for the spin-orbit separability of a laser beam, Phys. Rev. A 82, 033833 (2010).

[33] M. H. M. Passos, W. F. Balthazar, J. A. de Barros, C. E. R. Souza, A. Z. Khoury, and J. A. O. Huguenin, Classical analog of quantum contextuality in spin-orbit laser modes, Phys. Rev. A 98, 062116 (2018).

[34] A. G. de Oliveira, M. F. Arruda, W. C. Soares, S. P. Walborn, A. Z. Khoury, A. Kanaan, P. S. Ribeiro, and R. M. de Araújo, Phase conjugation and mode conversion in stimulated parametric down-conversion with orbital angular momentum: A geometrical interpretation, Brazilian Journal of Physics 49, 10 (2019).

[35] S.-X. Qian, Y. Li, L.-J. Kong, and C. Tu, Phase conjugation of vector fields by degenerate four-wave mixing in a Fedoped LiNbO3, Opt. Lett. 39, 4907 (2014).

[36] B. Y. Zel'Dovich, V. Popovichev, V. Ragul'Skii, and F. Faizullov, in Landmark Papers on Photorefractive Nonlinear Optics, edited by P. Yeh and C. Gu (World Scientific, Singapore, 1995), p. 303.

[37] M. D. Levenson, High-resolution imaging by wave-front conjugation, Opt. Lett. 5, 182 (1980).
[38] P. H. S. Souto Ribeiro, S. Pádua, and C. H. Monken, Image and coherence transfer in the stimulated down-conversion process, Phys. Rev. A 60, 5074 (1999).

[39] D. P. Caetano, M. P. Almeida, P. H. Souto Ribeiro, J. A. O. Huguenin, B. Coutinho dos Santos, and A. Z. Khoury, Conservation of orbital angular momentum in stimulated down-conversion, Phys. Rev. A 66, 041801(R) (2002).

[40] G. Milione, H. I. Sztul, D. A. Nolan, and R. R. Alfano, Higher-order Poincaré sphere, Stokes parameters, and the angular momentum of light, Physical Review Letters 107, 053601 (2011).

[41] G. Milione, S. Evans, D. A. Nolan, and R. R. Alfano, Higher Order Pancharatnam-Berry Phase and the Angular Momentum of Light, Phys. Rev. Lett. 108, 190401 (2012).

[42] D. M. Pepper, Nonlinear optical phase conjugation, Optical Engineering 21, 156 (1982).

[43] P. G. Kwiat, K. Mattle, H. Weinfurter, A. Zeilinger, A. V. Sergienko, and Y. Shih, New High-Intensity Source of Polarization-Entangled Photon Pairs, Phys. Rev. Lett. 75, 4337 (1995).

[44] T. Kim, M. Fiorentino, and F. N. C. Wong, Phase-stable source of polarization-entangled photons using a polarization Sagnac interferometer, Phys. Rev. A 73, 012316 (2006).

[45] M. F. Z. Arruda, W. C. Soares, S. P. Walborn, D. S. Tasca, A. Kanaan, R. Medeiros de Araújo, and P. H. Souto Ribeiro, Klyshko's advanced-wave picture in stimulated parametric down-conversion with a spatially structured pump beam, Phys. Rev. A 98, 023850 (2018).

[46] F. D. Martini, V. Buzek, F. Sciarrino, and C. Sias, Experimental realization of the quantum universal NOT gate, Nature 419, 815 (2002).

[47] F. DeMartini, D. Pelliccia, and F. Sciarrino, Contextual, Optimal, and Universal Realization of the Quantum Cloning Machine and of the NOT Gate, Phys. Rev. Lett. 92, 067901 (2004).

[48] S. P. Walborn, C. H. Monken, S. Pádua, and P. H. S. Ribeiro, Spatial correlations in parametric downconversion, Phys. Rep. 495, 87 (2010).

[49] J. Schneeloch and J. C. Howell, Introduction to the transverse spatial correlations in spontaneous parametric downconversion through the biphoton birth zone, J. Opt. 18, 053501 (2016).

[50] R. Rangarajan, M. Goggin, and P. Kwiat, Optimizing typeI polarization-entangled photons, Opt. Express 17, 18920 (2009).

[51] G. S. Agarwal and K. Tara, Nonclassical properties of states generated by the excitations on a coherent state, Phys. Rev. A 43, 492 (1991).

[52] M. V. Fedorov, M. A. Efremov, P. A. Volkov, E. V. Moreva, S. S. Straupe, and S. P. Kulik, Anisotropically and High Entanglement of Biphoton States Generated in Spontaneous Parametric Down-Conversion, Phys. Rev. Lett. 99, 063901 (2007).

[53] S. P. Walborn and C. H. Monken, Transverse spatial entanglement in parametric down-conversion, Phys. Rev. A 76, 062305 (2007).

[54] S. S. Straupe, D. P. Ivanov, A. A. Kalinkin, I. B. Bobrov, and S. P. Kulik, Angular Schmidt modes in spontaneous 
parametric down-conversion, Phys. Rev. A 83, 060302(R) (2011).

[55] S. P. Walborn, S. Pádua, and C. H. Monken, Conservation and entanglement of Hermite-Gaussian modes in parametric down-conversion, Phys. Rev. A 71, 053812 (2005).

[56] M. Suzuki, K. Yamane, K. Oka, Y. Toda, and R. Morita, Extended Stokes parameters for cylindrically polarized beams, Opt. Rev. 22, 179 (2015).

[57] G. Jaeger, Quantum Information An Overview (Springer, New York, 2007), 1st ed.

[58] L. J. Pereira, W. T. Buono, D. S. Tasca, K. Dechoum, and A. Z. Khoury, Orbital-angular-momentum mixing in typeII second-harmonic generation, Phys. Rev. A 96, 053856 (2017).
[59] W. T. Buono, J. Santiago, L. J. Pereira, D. S. Tasca, K. Dechoum, and A. Z. Khoury, Polarization-controlled orbital angular momentum switching in nonlinear wave mixing, Opt. Lett. 43, 1439 (2018).

[60] H.-J. Wu, B. Zhao, C. Rosales-Guzmán, W. Gao, B.-S. Shi, and Z.-H. Zhu, Spatial-Polarization-Independent Parametric Up-Conversion of Vectorially Structured Light, Phys. Rev. Appl. 13, 064041 (2020).

[61] J. T. Barreiro, T.-C. Wei, and P. G. Kwiat, Remote Preparation of Single-Photon "hybrid" Entangled and VectorPolarization States, Phys. Rev. Lett. 105, 030407 (2010).

[62] R. Jáuregui and J. P. Torres, On the use of structured light in nonlinear optics studies of the symmetry group of a crystal, Sci. Rep. 6, 20906 (2016). 